\title{
Diversity Analysis for Ensembles of Word Sequence Recognisers
}

\author{
Roman Bertolami and Horst Bunke \\ Institute of Computer Science and Applied Mathematics \\ University of Bern, Neubrückstrasse 10, CH-3012 Bern, Switzerland \\ \{bertolam, bunke\}@iam.unibe.ch
}

\begin{abstract}
In this paper we propose a general framework for analysing the diversity of ensembles of word sequence recognition systems. The goal of the framework is to enable the application of any diversity measure developed for standard multi-class classification problems to ensembles of word sequence recognisers. Experiments with several diversity measures are conducted on artificial as well as on real world data and show the effectiveness of the proposed approach.
\end{abstract}

\section{Introduction}

Ensemble methods have been applied to many different fields of pattern recognition [1. In handwriting recognition, improvements have been reported in isolated character 2 as well as in single word recognition 34. Only recently work has been published on ensemble methods for word sequence recognition [5]6], which is still a field with many challenges.

The goal of ensembles methods is to correct the errors of one ensemble member with the output of the other ensemble members. To achieve this goal we need a certain diversity among the ensemble members. Intuitively speaking, the members should make no coincident errors, i.e. the errors of one classifier should be independent of the errors of the other classifiers. A high diversity of a classifier ensemble is considered to be a strong hint to good performance. Hence, measuring diversity allows one to predict the performance of an ensemble without the need of conducting computationally expensive experiments. Several diversity measures have been proposed in the literature for multi-class problems. Surveys can be found in 8910].

Two different groups of diversity measures for ensembles of classifiers can be distinguished: pairwise measures and nonpairwise measures. Pairwise measures derive the final diversity of an ensemble of $N$ classifiers from the $N(N-1)$ pairwise diversity values. Usually the mean of these values is used as ensemble diversity. Popular members of this group of diversity measures are correlation, disagreement, and double fault. On the other hand, nonpairwise measures consider all the classifier of an ensemble together and calculate one diversity value directly. Popular nonpairwise diversity measures are entropy and Sharkey-Level based measures. 
All diversity measures currently known have been developed for conventional multi-class classification problems. To the knowledge of the authors, no diversity measures for word sequence recognition have been proposed until know.

The aim of the current paper is to provide a generic framework to apply diversity measures, developed for conventional multi-class classification problems, to the task of word sequence recognition. Word sequence recognition is a difficult problem because not only a single class, but a sequence of word classes of unknown length has to be returned by the recogniser. Even though appropriate diversity measures for word sequence recognition are potentially useful in the ensemble generation process, no such measures have been published yet. The contribution of this paper is to make classical diversity measures available for word sequence recognisers.

The remaining part of the paper is organised as follows. Section 2 introduces the diversity analysis framework and provides an example in detail. Experiments conducted on artificial as well as on real world data are described in Sect. 3 . Finally, conclusions are drawn in the last section of the paper.

\section{Methodology}

Assume we have an ensemble where each of the $n$ recognisers outputs a word sequence $W_{i}=\left(w_{i_{1}}, \ldots, w_{i_{m_{i}}}\right) ; i=1, \ldots, n$. The number of words $m_{i}$ in these sequences may differ and therefore a synchronisation process is required first. The synchronised results of the ensemble members are stored in a Word Transition Network (WTN) [11. Because the class labels are used to calculate the diversity measures, the segments of the WTN have to be labelled with the ground truth. Any diversity measure available for multi-class problems can then be applied to the segments and based on these measures the final ensemble diversity is derived. Next, we describe various aspects of the proposed diversity analysis framework in greater detail. An extensive example of the entire process is provided in Section 2.3 .

\subsection{Synchronisation of the Word Sequences}

Let the $n$ ensemble members have recognised their individual word sequences $\left(W_{1}, \ldots, W_{n}\right)$. Each of these sequences might contain a different amount of words and therefore an alignment procedure is necessary to synchronise the $n$ word sequences. Any possible string alignment procedure can be used for this purpose. However, because the optimal alignment of multiple strings is an $N P$ complete problem [12, we suggest to use a heuristic approach (e.g. incremental) for the alignment. For details see 11. The result of the alignment is a WTN which consists of $m$ segments. Each arc in the WTN is labelled with a word out of $\left(W_{1}, \ldots, W_{n}\right)$. Null transition arcs $\varepsilon$ occur when the number of words in $\left(W_{1}, \ldots, W_{n}\right)$ is not equal for each $W_{i}(i=1, \ldots, n)$.

Next, the ensemble result is derived applying some decision rule to each segment of the WTN. Any kind of decision strategy can be used, but for the sake 
Table 1. Probabilities of coincident errors between classifier $C_{i}$ and $C_{j}$

\begin{tabular}{c|c|c} 
& $C_{i}$ correct & $C_{i}$ wrong \\
\hline$C_{j}$ correct & $a$ & $b$ \\
$C_{j}$ wrong & $c$ & $d$
\end{tabular}

of simplicity we just use a voting procedure. The resulting sequence of decision results constitute the combination result $\hat{W}$. Note that if the decision result of a segment is a null transition, this segment does not contribute any word to $\hat{W}$.

Once we have the combination result $\hat{W}$ and the ground truth, we can label the segments of the WTN. Therefore, we first align $\hat{W}$ with the ground truth. Using the alignment we can map the words in the ground truth to the WTN segments.

\subsection{Application of Diversity Measures}

Existing diversity measures can then be applied to the labelled segments of the WTN, similarly to conventional multi-class classification problems. The average of these measures gives the final ensemble diversity.

In the present work we apply pairwise as well as Sharkey-Level based diversity measures to word sequence recognition. Pairwise diversity measures consider only a pair of recognisers at a time. Any possible pair of ensemble members produces a diversity value. The average across all pairs gives the final diversity. Based on the probabilities of coincident errors between two recognisers $C_{i}$ and $C_{j}$ (Tab. 1) several measures have been proposed.

Correlation. Because the output of two recognisers can be considered as numerical values ( 1 for correct and 0 for wrong), we can calculate the correlation coefficients.

$$
\rho_{i, j}=\frac{a d-b c}{\sqrt{(a+b)(c+d)(a+c)(b+d)}}
$$

Disagreement. The disagreement measure is a very intuitive measure of diversity. It is the probability that the two considered recognisers will disagree on their decision.

$$
D_{i, j}=b+c
$$

Double Fault. Another quite intuitive measure is the double fault measure which is the probability that both recognisers make a wrong decision.

$$
D F_{i, j}=d
$$

The second kind of diversity measures are derived from the Sharkey-Levels introduced in 13. Each segment of the WTN can be assigned to one of the following levels:

Level 1. No coincident errors. Each ensemble member produces the correct result. 
Level 2. Some coincident errors, but the majority of the ensemble members provide the correct result.

Level 3. Majority is not correct but some of the members produce the correct result.

Level 4. The correct result is not output by any of ensemble members.

The frequencies of the different levels are then used as diversity measures. Thus, $L_{i}$ is the frequency that a segment of the WTN belongs to Level $i$, where $i=1, \ldots, 4$.

\subsection{Example}

Next, we will provide an example of the entire process of calculating diversity measures for ensembles of handwritten text line recognisers. The input is the handwritten text line They will be asked to comment shown in Fig 1. Features are extracted and each ensemble member performs the recognition step. The recognised word sequences $\left(W_{1}, \ldots, W_{9}\right)$ are shown in Fig. 2 ,

Next, the word sequences $\left(W_{1}, \ldots, W_{9}\right)$ are synchronised. An iterative alignment is used for this purpose [11]. The result of this alignment step is shown in Fig. 3. Note that null transition arcs have to be inserted at the beginning of some text lines to align the additional word in $W_{1}$ and $W_{7}$.

Once the alignment is performed, we can calculate the combination result for each alignment segment. We apply a majority voting and get the word sequence they will be asked to council. To label the alignment segments we first have to align the combination result $(\hat{W})$ with the ground truth $(\mathrm{T})$ as shown in Fig. 4 ,

Based on this information we label the segments of the aligned word sequences of Fig. 3. The result of the labelling process is shown in Fig. 5.

Now we are able to apply any of the described diversity measures, originally developed for conventional multi-class classification problems, to our sequence recognition problem. E.g. the Level 1 diversity measure yields 4/7, whereas the Level 3 diversity measure is equal to $1 / 7$.

\section{Experiments and Results}

One of the main motivations for computing ensemble diversity is to predict the performance of an ensemble of recognisers without the need to to run computationally expensive experiments. Hence, the aim of the experiments is to show relationships between the recognition accuracy and the different diversity measures. Experimental evaluation of the proposed framework is conducted on a synthetic and a real world data set.

\subsection{Synthetic Word Sequences}

To be able to test our framework, we generate artificial ensemble results. First the ground truth is created and then the ensemble results are generated. 


\section{They will
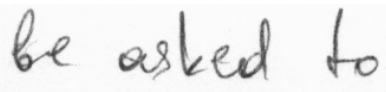 \\ comment}

Fig. 1. Handwritten input text

$W_{1}$ : if they will be asked to council

$W_{2}$ : they will be asked to comment

$W_{3}$ : it will be asked to comment

$W_{4}$ : they will be asked to council

$W_{5}$ : they will be asked to council

$W_{6}$ : they will be asked to comment

$W_{7}$ : if it will be asked to comment

$W_{8}$ : they will be asked to council

$W_{9}$ : they will be asked to council

Fig. 2. Results from the different ensemble members

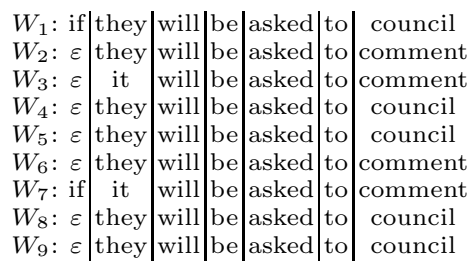

Fig. 3. Alignment of the ensemble results in a WTN

$\hat{W}$ : they |will|be asked to council

$\mathrm{T}$ : They $\mid$ will $\mid$ be $\mid$ asked $\mid$ to comment

Fig. 4. Alignment of the combination result and the ground truth

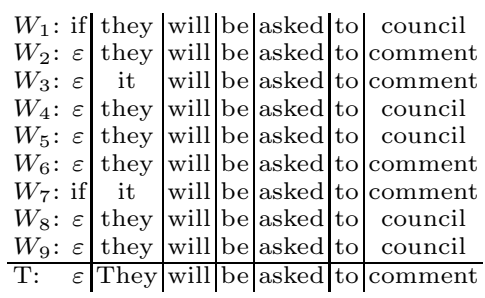

Fig. 5. Labelling the segments

To build the ground truth for a word sequence, the number of words $n$ is first defined by a random number in a given range. Next, $n$ words are randomly chosen from the underlying lexicon and then used as the ground truth word sequence. 
Next, the ensemble results are generated iteratively. Given the ground truth word sequence, the first ensemble member's result sequence is created randomly with a given accuracy. The next member's result sequence is then generated with respect to a defined correlation to the previously generated word sequence. This process is continued until the desired number of results has been obtained.

We generated ensembles with five, ten, fifteen, and twenty members with a lexicon of 10,000 word classes. In our experiments we also varied the correlation between two successive members to obtain ensembles with different diversities.

The advantage of the artificial data is that we can control the correlation between the ensemble members. Also the number of classes can be chosen. Therefore, we are able to simulate different application domains, e.g. character sequences $(\sim 80$ classes $)$ or word sequences $(\sim 10,000$ classes $)$. Furthermore, an arbitrarily large amount of data can be produced relatively fast. On the other hand, it may happen that the generated ensemble member results do not sufficiently well model the results produced by real word ensemble members.

\subsection{Ensembles of Handwritten Text Line Recognisers}

In the second part of the experimental evaluation we use real word data from offline handwritten text line recognition. The data originate from [14] where three different ensemble member selection strategies have been validated. For this purpose, many different ensembles of various sizes have been built and tested which we now use to evaluate the proposed diversity framework.

The ensemble members were derived from specific integration of a statistical language model in the hidden Markov model based recognition system. The handwritten text lines that were used originate from the IAM 1 database [15]. For further details about the experimental setup of the recognition and ensemble generation step we refer to 14 .

\subsection{Diversity Analysis Results}

In the experiments described in this section we investigate which of the proposed diversity measures, applied in our framework, is a better indicator of the accuracy of the combined ensemble results. Furthermore, we analyse the differences between the artificial and the real world data results.

The results for the pairwise measures Correlation, Disagreement, and Double Fault are shown in Fig. 6. While clear tendencies can be seen in the outcomes of the artificial ensemble, only the Double Fault measure seems to be a useful indicator for the accuracy of the ensemble performance on the real world data. The four different lines that can be observed in the artificial data plots originate from the four different sizes of the analysed ensembles.

Figure 7 shows the results of the level based diversity measures. On the real world data set, the best indicator of a good performance is Level 4. This means

\footnotetext{
${ }^{1}$ The IAM database is publicly available for download at http://www.iam.unibe.ch/ $\sim \mathrm{fki} / \mathrm{iamDB}$
} 

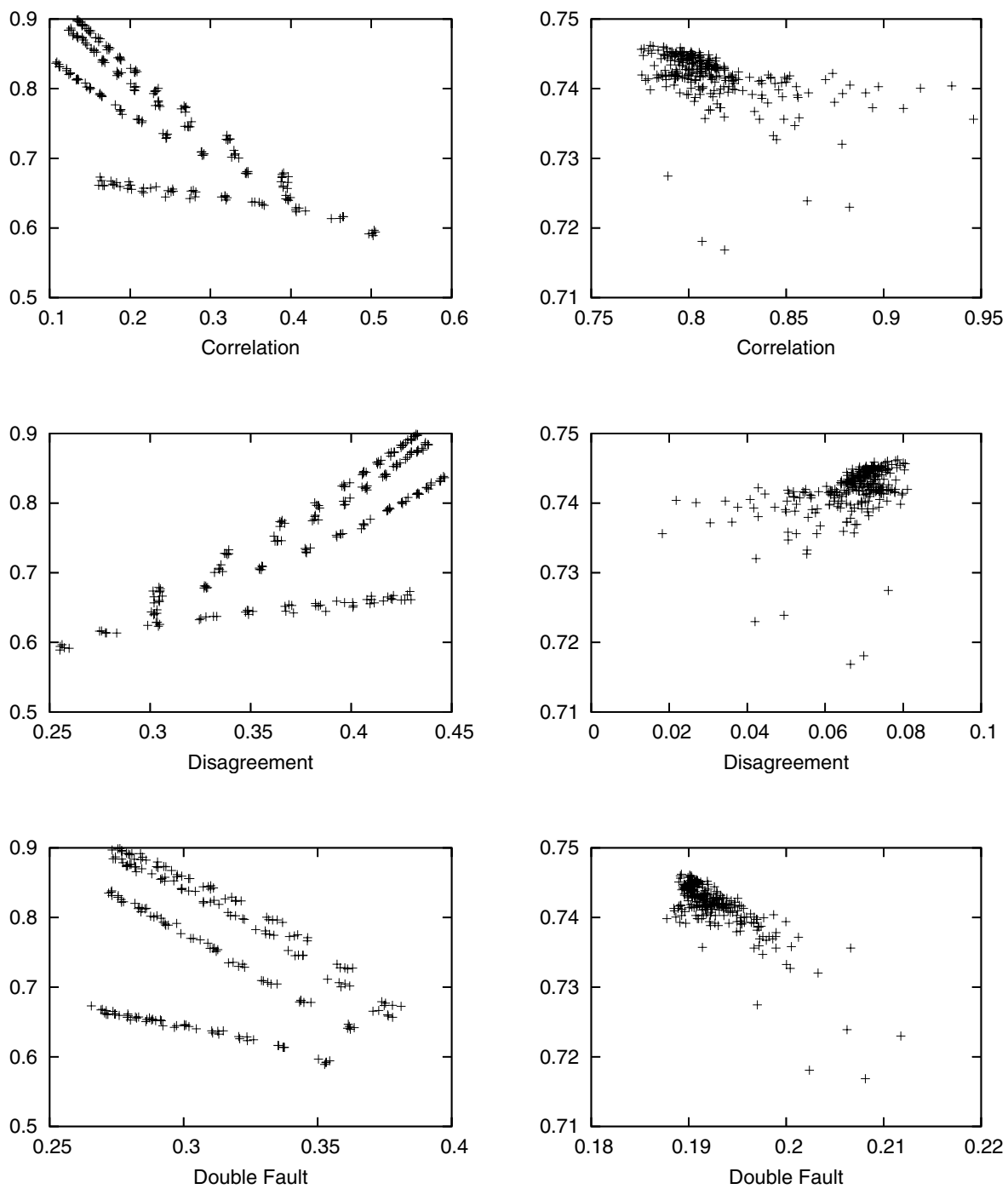

Fig. 6. Evaluation of pairwise diversity measures. The x-axis shows the values of the diversity measure whereas the ensemble accuracy is displayed on the y-axis. The results with artificial data are shown in left column, whereas the outcome on the real world data is shown on the right.

that if we are able to decrease the number of segments where the target word does not even occur a single time, we can expect that the overall performance of the ensemble increases.

The correlation coefficients between the different diversity measures and the recognition accuracy are listed in Tab 2. These values support the optical impression of Fig. 6 and Fig. 7 

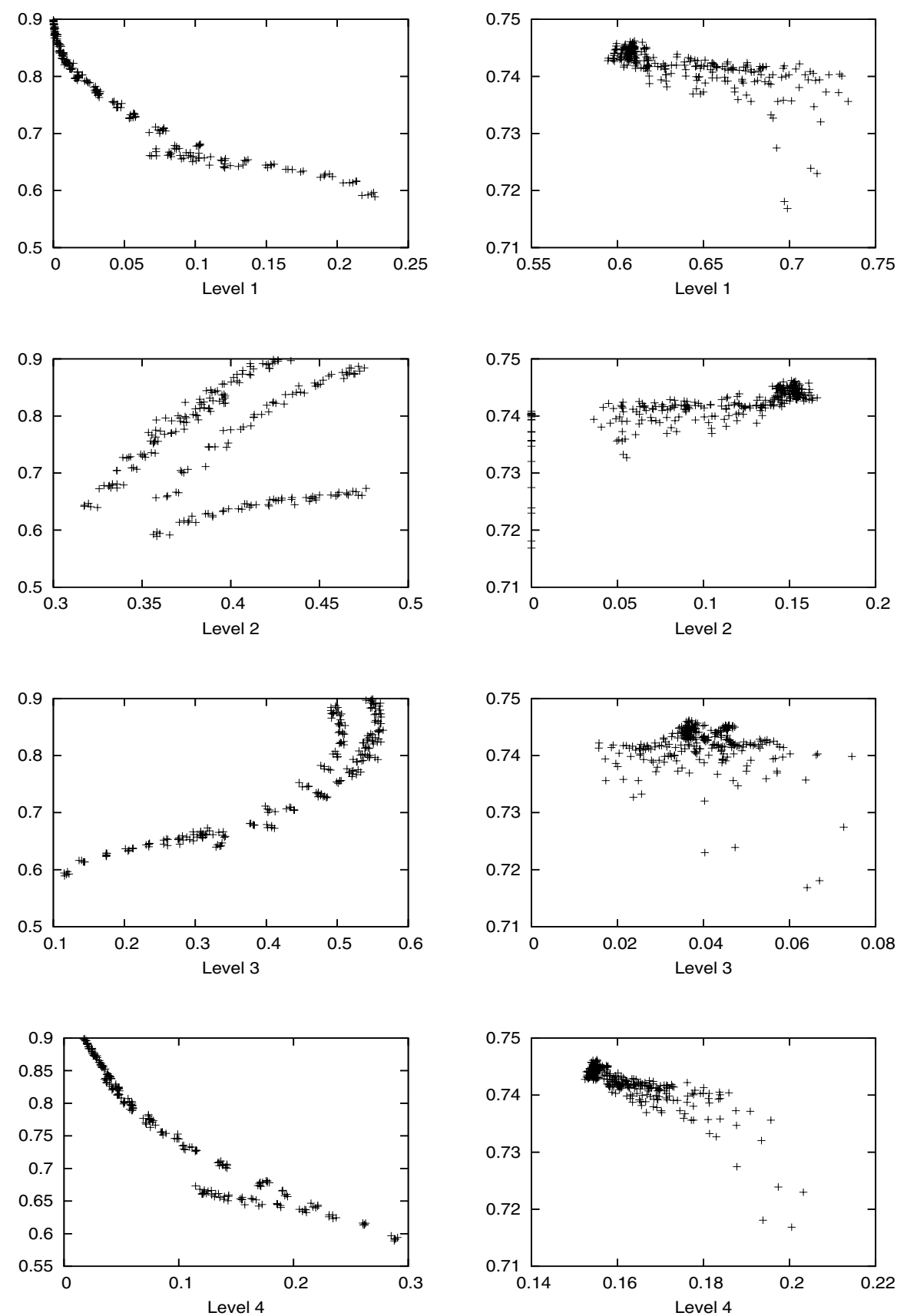

Fig. 7. Evaluation of level based diversity measures. The $x$-axis shows the values of the diversity measure whereas the ensemble accuracy is displayed on the y-axis. The results with artificial data are shown in left column, whereas the outcome on the real world data is shown on the right. 
Table 2. Correlation coefficients of the different diversity measures

\begin{tabular}{l|c|c} 
& Artificial Data & Real Data \\
\hline Correlation & -0.8 & -0.45 \\
Disagreement & 0.77 & 0.42 \\
Double Fault & -0.45 & -0.81 \\
\hline Level 1 & -0.92 & -0.66 \\
Level 2 & 0.3 & 0.7 \\
Level 3 & 0.9 & -0.15 \\
Level 4 & -0.95 & -0.83
\end{tabular}

\section{Conclusions}

We have proposed a framework for diversity analysis of ensembles of word sequence recognition systems. The framework allows one to apply any diversity measure available for conventional multi-class classification problems to ensembles of word sequence recognisers.

In the proposed framework, the word sequences of the individual ensemble members are synchronised in a sequence of segments first. Next, these segments are labelled with the ground truth. Once each segment is labelled, diversity measures for multi-class problems can be applied to the segments. The average of these values gives then the final diversity of the ensemble.

Experiments have been conducted with artificial as well as with real world data from offline handwritten text line recognition. Several pairwise and nonpairwise diversity measures have been applied to both tasks to show the effectiveness of the different measures within the proposed framework. Some of these diversity measures seem to be useful indicators of good ensemble performance.

In the current paper we have exclusively considered the task of word sequence recognition. However, the proposed framework is applicable to continuous speech recognition and other domains as well, where an individual classifier outputs a sequence of classes, rather than just a single class.

\section{Acknowledgement}

This research was supported by the Swiss National Science Foundation (Nr. 200020-19124/1). Additional funding was provided by the Swiss National Science Foundation NCCR program "Interactive Multimodal Information Management (IM)2" in the Individual Project "Visual/video processing".

\section{References}

1. Oza, N., Polikar, R., Kittler, J., Roli, F., eds.: Multiple Classifier Systems, 6th International Workshop, Springer LNCS 3541 (2005)

2. Huang, T., Suen, C.: Combination of multiple experts for the recognition of unconstrained handwritten numerals. IEEE Transactions on Pattern Analysis and Machine Intelligence 17 (1995) 90-94 
3. Gader, P., Mohamed, M., Keller, J.: Fusion of handwritten word classifiers. Pattern Recognition Letters 17 (1996) 577-584

4. Günter, S., Bunke, H.: Ensembles of classifiers for handwritten word recognition. International Journal on Document Analysis and Recognition 5 (2003) $224-232$

5. Nakano, Y., Hananoi, T., Miyao, H., Maruyama, M., Maruyama, K.: A document analysis system based on text line matching of multiple ocr outputs. In: Document Analysis Systems VI, 6th International Workshop, Florence, Italy. (2004)

6. Wilczok, E., Lellmann, W.: Adaptive combination of commercial OCR systems. In Andreas Dengel, Markus Junker, A.W., ed.: Reading and Learning: Adaptive Content Recognition, Springer-Verlag Heidelberg (2004) 124-136

7. Bertolami, R., Bunke, H.: Multiple handwritten text recognition systems derived from specific integration of a language model. In: 8th International Conference on Document Analysis and Recognition, Seoul, Korea. Volume 1. (2005) 521-524

8. Windeatt, T.: Diversity measures for multiple classifier system analysis and design. Information Fusion 6 (2004) 21-36

9. Brown, G., Wyatt, J., Harris, R., Yao, X.: Diversity creation methods: A survey and categorisation. Information Fusion 6 (2005) 5-20

10. Kuncheva, L.I.: Combining Pattern Classifiers: Methods and Algorithms. John Wiley \& Sons Inc (2004)

11. Fiscus, J.: A post-processing system to yield reduced word error rates: Recognizer output voting error reduction. In: IEEE Workshop on Automatic Speech Recognition and Understanding (ASRU), Santa Barbara. (1997) 347-352

12. Wang, L., Jiang, T.: On the complexity of multiple sequence alignment. Journal of Computational Biology 1 (1994) 337-348

13. Sharkey, A., Sharkey, N.: Combining diverse neural networks. The Knowledge Engineering Review 12 (1997) 231-247

14. Bertolami, R., Bunke, H.: Ensemble methods for handwritten text line recognition systems. In: International Conference on Systems, Man and Cybernetics, Hawaii, USA. (2005) 2334-2339

15. Marti, U.V., Bunke, H.: The IAM-database: an English sentence database for offline handwriting recognition. International Journal on Document Analysis and Recognition 5 (2002) $39-46$ 